\title{
Applied basic science in process analytics and control technology
}

\section{Christoph Herwig ${ }^{1}$}

Published online: 13 February 2020

(C) Springer-Verlag GmbH Germany, part of Springer Nature 2020

Process analytical technology is a system for designing, analyzing, and controlling manufacturing through timely controlled measurements of critical quality and performance attributes with the goal of ensuring final product quality (ICH Q8R2). This definition is more than 10 years old but is currently of high interest.

When we interpret this definition, we see a three-column concept: to use PAT to design, analyze, and control manufacturing processes. Hence, PAT is much more than just a measurement in the process. PAT is a multifold and multidisciplinary topic. We need

(1) new scientific approaches to design sensors which have high specificity and resolution (= data),

(2) to make sure we can contextualize, aggregate, fuse, and manage the data from PAT devices (= information)

(3) to achieve process understanding (= knowledge), which is the core of the overarching initiative of Quality by Design (QbD), while we can use digital twins as an excellent tool for capturing and managing knowledge

(4) to deploy the knowledge to render benefits in its applications using control technology (= wisdom).

So, what are the applications of the future, which will need process analytics and control technology (PACT)?

Published in the topical collection Advances in Process Analytics and Control Technology with guest editor Christoph Herwig.

Christoph Herwig

christoph.herwig@tuwien.ac.at

1 Institute of Chemical, Environmental and Bioscience Engineering, Research Area Biochemical Engineering, Technische Universität Wien, Gumpendorfer Straße 1a, 1060 Vienna, Austria
The current hype on digital transformation by Internet of things (IoT) will need a smart integration of PAT as essential data source and the backbone of domain knowledge.

Especially in biotechnological market, we also need, finally, tools to optimize processes due to the increased competition in biosimilars as well as to counteract drug shortages and provide affordable drugs to the world.

Therefore, we

- need to be quicker in time to clinic by PACT-based experimental design,

- have a full usage of the installed assets by turning to continuous process modes by using PACT-based controls and

- be right first time in advanced therapeutic medicinal products (ATMPs) because your blood is different from mine by using data science-driven PACT-based platform knowledge.

Hence, PACT will be the key enabler for robustifying continuous bioprocessing and advanced therapies. To be successful, we need a scientific approach that combines basic science with solutions, which are ready to be transferred to application.

In this topical collection on Process Analytics and Control Technology, we want to highlight the recent advances in PACT addressing research activities, critical reviews on recent developments, and extrapolating to likely future trends.

This issue starts with two feature articles describing future requirements for smart labs and smart sensors, in which PACT will play a key role. A critical review is included to elucidate potentials and gaps for PAT in downstream bioprocessing. In the research papers, we will show novel sensors and miniaturized sensors for process monitoring, which are beyond current spectroscopic solutions. We show how PAT data can be aggregated in soft sensors and kinetic models for enhance 
process transparency and their use in process design and process control.

If you want to see more of those current trends and want to discuss them in the scientific community, please join the European Conferences on Process Analytics and Control Technology, organized every three years by DECHEMA (Gesellschaft für Chemische Technik und Biotechnologie e.V.).

Acknowledgments I would like to thank all authors for submitting their valuable work for this topical collection. I would also like to acknowledge the contributions of referees. It would not be possible to achieve such a high quality without their careful review and constructive criticisms. Finally, I would like to thank the Editorial Office and Editors for their friendly and timely support.

Publisher's note Springer Nature remains neutral with regard to jurisdictional claims in published maps and institutional affiliations.

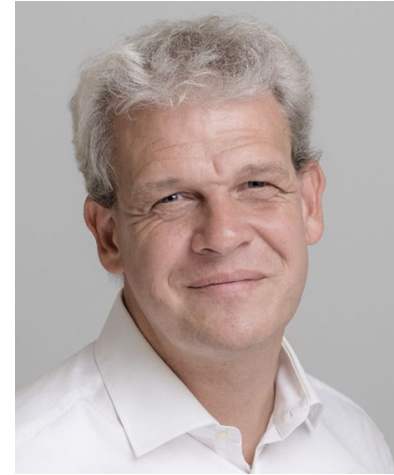

Christoph Herwig is a bioprocess engineer from RWTH Aachen, who worked in the industry in the design and commissioning of large chemical facilities prior to entering his interdisciplinary $\mathrm{PhD}$ studies at EPFL, Switzerland, in bioprocess identification. Subsequently, he positioned himself at the interface between bioprocess development and facility design in biopharmaceutical industry. Since 2008, he has been a full professor for biochemical engineering at the Vienna University of Technology. His research area focuses on the development of data science methods for integrated and efficient bioprocess development along PAT and QbD principles. The product fields are circular economy and biopharmaceuticals within industry-driven projects. In 2013, he founded the company Exputec, addressing data science solutions for the biopharma life cycle. Currently, he is the chairman of the board of the Working Group Process Analytics (Arbeitskreis Prozessanalytik) of the GDCh and Dechema. 